\title{
Intronic sequences are required for AINTEGUMENTA-LIKE6 expression in Arabidopsis flowers
}

Beth A. Krizek * (D)

\begin{abstract}
Background: The AINTEGUMENTA-LIKE6/PLETHORA3 (AIL6/PLT3) gene of Arabidopsis thaliana is a key regulator of growth and patterning in both shoots and roots. AlL6 encodes an AINTEGUMENTA-LIKE/PLETHORA (AIL/PLT) transcription factor that is expressed in the root stem cell niche, the peripheral region of the shoot apical meristem and young lateral organ primordia. In flowers, AIL6 acts redundantly with AINTEGUMENTA (ANT) to regulate floral organ positioning, growth, identity and patterning. Experiments were undertaken to define the genomic regions required for All6 function and expression in flowers.

Results: Transgenic plants expressing a copy of the coding region of All 6 in the context of $7.7 \mathrm{~kb}$ of $5^{\prime}$ sequence and 919 bp of $3^{\prime}$ sequence (AlL6:CAlL6-3) fail to fully complement AlL6 function when assayed in the ant-4 ail6-2 double mutant background. In contrast, a genomic copy of AlL 6 with the same amount of $5^{\prime}$ and $3^{\prime}$ sequence (AlL6:gAll6-31) can fully complement ant-4 ail6-2. In addition, a genomic copy of All 6 with $590 \mathrm{bp}$ of $5^{\prime}$ sequence and $919 \mathrm{bp}$ of $3^{\prime}$ sequence (AlL6m:gAlL6-3) complements ant-4 ail6-2 and contains all regulatory elements needed to confer normal AlL6 expression in inflorescences. Efforts to map cis-regulatory elements reveal that the third intron of AlL 6 contains enhancer elements that confer expression in young flowers but in a broader pattern than that of AlL6 mRNA in wildtype flowers. Some AlL6:gAlL6-3' and AlL6m:gAlL6-3' lines confer an over-rescue phenotype in the ant-4 ail6-2 background that is correlated with higher levels of AlL6 mRNA accumulation.
\end{abstract}

Conclusions: The results presented here indicate that AlL 6 intronic sequences serve as transcriptional enhancer elements. In addition, the results show that increased expression of AlL 6 can partially compensate for loss of ANT function in flowers.

Keywords: AINTEGUMENTA-LIKE6 (AIL6), AINTEGUMENTA-LIKE/PLETHORA (AIL/PLT) family, AINTEGUMENTA (ANT), Flower development, Arabidopsis thaliana, Transcription factors, Enhancers, Gene regulation

\section{Background}

AIL6 encodes a member of the small subfamily of AIL/ PLT transcription factors that are part of the large AP2/ ERF family in Arabidopsis thaliana [1]. AIL proteins are key regulators of developmental processes throughout the plant life cycle (reviewed in [2]). AIL6 regulates multiple processes during Arabidopsis root and shoot development, largely in a redundant fashion with other

*Correspondence: krizek@sc.edu

Department of Biological Sciences, University of South Carolina, Columbia, SC 29208, USA
AIL genes. Loss of AIL6 function on its own has no obvious phenotype in the shoot and only results in a slightly shorter root and root apical meristem [3, 4]. Within shoots, AIL6 acts with ANT and AINTEGUMENTALIKE7/PLETHORA7 (AIL7/PLT7) to maintain the shoot apical meristem during vegetative development and works in a redundant fashion with AINTEGUMENTALIKE5/PLETHORA5 (AIL5/PLT5) and AIL7 to control shoot phyllotaxy [5, 6]. In flowers, AIL6 acts redundantly with $A N T$ to regulate floral organ initiation, growth, identity specification and patterning [4]. AIL6 function is required for root formation in combination with 
PLETHORA1 (PLT1) and PLETHORA2 (PLT2) and controls the positioning of lateral roots in a redundant fashion with AIL5 and AIL7 [3, 7].

As expected from its functions throughout the plant, AIL6 is expressed at the mRNA level in multiple tissues. In roots, AIL6 mRNA is detected in the stem cell niche, pericycle cells prior to lateral root initiation, and lateral root primordia [3, 7]. In the shoot, AIL6 mRNA is detected in young lateral organ primordia and throughout the shoot apical meristem with expression higher in the periphery of the meristem and incipient lateral organ as compared with the center of the meristem $[1,5]$. In flowers, AIL6 expression is associated with young flower primordium and early stages of floral organ development. AIL6 is expressed throughout stage one and two flower primordia, becoming primarily restricted to the floral meristem dome in stage three flowers with only low levels present in sepal primordia [1]. In stage six flowers, some AIL6 mRNA is present within petal, stamen and carpel primordia; only low amounts of AIL6 mRNA are detected after this stage of development [1].

In several of these tissues, AIL6 expression is linked with the activity of AUXIN RESPONSE FACTORs (ARFs), transcription factors that regulate gene expression in response to auxin. In lateral roots, AIL6 appears to act downstream of ARF7 and ARF19 [7]. AIL6 is not expressed in any pericycle cells of arf7 arf19 double mutants, which lack most lateral roots, although it is not known whether AIL6 is a direct target of these transcription factors [7]. Chromatin immunoprecipitation (ChIP) experiments show that MONOPTEROS/AUXIN RESPONSE FACTOR 5 (MP/ARF5) directly activates AIL6 in the periphery of the shoot apical meristem to promote flower primordium initiation [8]. Two other potential regulators of AIL6 are the floral meristem identity protein LEAFY (LFY) and APETALA1 (AP1) which were shown to bind to AIL6 in genome-wide ChIP experiments $[9,10]$.

Despite the importance of AIL6 in Arabidopsis vegetative and reproductive development, sequences required for proper AIL6 expression have not been identified. Here I define the genomic regions necessary for AIL6 function in flowers by complementation of ant-4 ail6-2 double mutants with transgenes containing different amounts of AIL6 sequence. These experiments show that introns are required for AIL6 function and expression in flowers. In particular, intron three was found to contain enhancer elements that drive AIL6 expression in early stages of flower development. The importance of intron three to AIL6 regulation is also demonstrated by work from other labs showing binding of LFY and AP1 to the third intron of AIL6 [9, 10]. Furthermore, this work demonstrates that increased expression of AIL6 can partially compensate for loss of $A N T$ function.

\section{Results and discussion}

\section{Intronic sequences are required for complementation} of AIL6 function in ant ail6 flowers

To define the genomic regions required for AIL6 function in flower development, ant-4/+ ail6-2 plants were transformed with a transgene containing the AIL6 coding region in a genomic context of $7.7 \mathrm{~kb}$ of $5^{\prime}$ sequence and 919 bp of 3' sequence (i.e. AIL6:CAIL6-3'; Fig. 1a). The ant-4 ail6-2 double mutant was used in these studies since ail6-2 single mutants show no flower phenotype [4]. Of the six transgenic lines obtained, three lines (lines 2 , 9, and 10) showed a partial rescue of the ant-4 ail62 double mutant phenotype while three lines (lines 11, 14 and 16) exhibited no rescue. In the partially rescued AIL6:CAIL6-3' ant-4 ail6-2 line 2, the flowers are intermediate in severity between those of ant-4 and those of ant-4 ail6-2 (Fig. 2a-d). They are larger than those of ant-4 ail6-2 and consist primarily of sepals, filaments, stamens and carpel valves fused to varying extents (Fig. 2d; Table 1). In contrast, ant-4 ail6-2 flowers consist of sepals, filaments, stamenoid organs and unfused carpel valves (Fig. 2c; Table 1). The stamens in AIL6:cAIL6-3' ant-4 ail6-2 line 2 flowers resemble those of ant-4 with two locules rather than the four locules present in wildtype stamens, and some produce pollen. No petals were produced in these flowers. The leaves of AIL6:CAIL6-3' ant-4 ail6-2 line 2 were similar in size to those of ant-4 ail6-2 in 20 day old plants (Fig. $2 \mathrm{~g}-\mathrm{j}$ ).

To determine whether the lack of complementation by the AIL6:CAIL6-3' transgene was a consequence of the absence of introns or insufficient $5^{\prime}$ and $3^{\prime}$ sequence, ant-4/+ ail6-2 plants were transformed with a transgene corresponding to a genomic copy of AIL6 in the same context of $5^{\prime}$ and $3^{\prime}$ sequence (AIL6:gAIL6-3) (Fig. 1a). Of the 5 lines obtained, one line (line 12) exhibited partial rescue of ant-4 ail6-2, two lines (lines 11 and 17) exhibited complete rescue such that the flowers resembled ant-4, and two lines (lines 4 and 21) exhibited an overrescue phenotype such that the plants had a less severe phenotype than ant-4 (Fig. 2e, f). The phenotypic variation in the degree of complementation in these lines is presumably a consequence of variation in transgene insertion sites. AIL6:gAIL6-3' ant-4 ail6-2 line 17 flowers closely resemble ant- 4 flowers with regard to the identity, numbers, and size of the floral organs (Fig. 2e; Table 1). In flowers of the over-rescue AIL6:gAIL6-3' ant-4 ail6-2 line 4, the petals are larger than those of ant-4 and the stamen anthers consist of four locules (Fig. 2f). However, AIL6:gAIL6-4' ant-4 ail6-2 line 4 petals are not as big as 


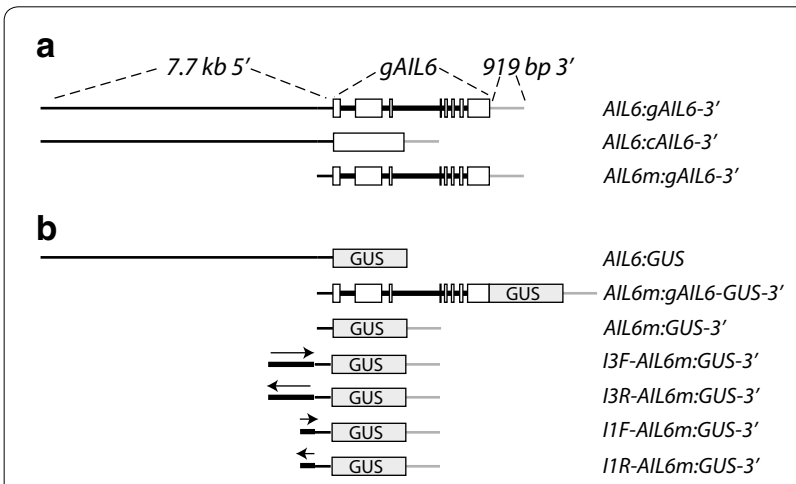

Fig. 1 All 6 constructs used in this study. a Constructs tested for complementation of AlL 6 function in the ant-4 ail 6 - 2 double mutant background. AlL6 5' sequences are shown with thin black lines, exons with white boxes, introns with thick black lines, and AlL6 $3^{\prime}$ sequences with gray lines. CAlL6 corresponds to the coding region of AlL6. AlL6m corresponds to $590 \mathrm{bp}$ of $5^{\prime}$ sequence directly upstream of the start codon. b GUS reporter constructs. AlL6 5' sequences are shown with thin black lines, exons with white boxes, introns with thick black lines, and AlL6 $3^{\prime}$ sequences with gray lines. Arrows show the direction (Forward, F; or Reverse, $R$ ) for introns three (I3) and one (I1) in the reporter constructs

those of wild-type flowers, and the flowers are female sterile and consist of fewer petals and stamens compared to wild-type (Fig. 2f; Table 1). Similar effects were observed with regard to complementation of leaf growth defects in AIL6:gAIL6-3' ant-4 ail6-2 lines 17 and 4; line 17 leaves resemble those of ant-4 while line 4 leaves are larger (Fig. 2k, l, m).

\section{AlL6 mRNA accumulation depends on intronic sequences}

To determine whether the distinct phenotypes conferred by the AIL6:CAIL6-3' and AIL6:gAIL6-3' transgenes correlated with the levels of AIL6 mRNA accumulation in these plants, AIL6 mRNA levels were examined by reverse transcriptase quantitative PCR (RT-qPCR). AIL6:cAIL6-3' ant-4 ail6-2 line 2 inflorescences accumulate less AIL6 mRNA compared with Ler (Fig. 2n). AIL6:gAIL6-3' ant-4 ail6-2 lines 17 and 4 accumulate more AIL6 mRNA than Ler, approximately 2.3 and 6.5 fold higher levels than Ler, respectively (Fig. 2n). Since the ant-4 ail6-2 double mutant is in a mixed Ler/ Col background (see "Methods"), I also examined AIL6 mRNA levels in Col and found them to be similar to those in Ler (Fig. 2n). AIL6 mRNA levels were slightly increased in the ant-4 background, suggesting possible cross-regulation of AIL6 expression. The increased levels of AIL6 mRNA in lines 17 and 4 compared to Ler, Col and ant-4 is likely a consequence of the chromosomal position of the transgene insertion site. The RT-qPCR results indicate that intronic sequences increase steady-state AIL6 mRNA levels. They also suggest that the inability of
AIL6:CAIL6-3' to fully complement AIL6 function in the ant-4 ail6-2 double mutant results from insufficient AIL6 mRNA. Furthermore, the higher AIL6 mRNA levels in AIL6:gAIL6-3' ant-4 ail6-2 line 4 as compared with line 17 suggest that increased $A I L 6$ activity can partially compensate for loss of $A N T$ function.

The spatial distribution of AIL6 mRNA in Ler and AIL6:gAIL6-3' ant-4 ail6-2 lines 17 and 4 was examined by in situ hybridization. Within the inflorescence meristem, AIL6 mRNA accumulates in a similar spatial pattern in Ler and AIL6:gAIL6-3' ant-4 ail6-2 lines 17 and 4 , with expression higher in the periphery of the meristem compared with the center (Fig. 3a, e). AIL6 mRNA accumulates throughout stage 1 and 2 flower primordia on the flanks of the inflorescence meristem in both Ler and AIL6:gAIL6-3' ant-4 ail6-2 lines 17 and 4 (Fig. 3a, f). AIL6 mRNA was detectable within sepal primordia of AIL6:gAIL6-3' ant-4 ail6-2 line 4 stage 4, 6 and 7 flowers, while sepals of stage 4 and 6 Ler flowers have no detectable AIL6 mRNA (Fig. 3b, c, g). Little AIL6 mRNA is detected in any floral organ after stage 6 of flower development (Fig. 3a-c). AIL6 was expressed in the procambium in AIL6:gAIL6-3' ant-4 ail6-2 lines 17 and 4 at noticeable higher levels than in Ler (Fig. 3a, e-g). This may in part be due to the ant-4 ail6-2 background, as AIL6 mRNA was also more easily detected in the procambium of ant-4 ail6-2 double mutants compared to Ler (Fig. 3d). In summary, the AIL6 mRNA expression pattern in AIL6:gAIL6-3' ant-4 ail6-2 line 17 flowers closely resembles that of Ler, while AIL6 mRNA is detected in a broader pattern in AIL6:gAIL6-3 ant-4 ail62 line 4 stage 4 flowers and persists longer in developing floral organs.

\section{Complementation of AIL6 function in ant ail 6 flowers by a smaller genomic fragment}

To refine the amount of $5^{\prime}$ sequence required for AIL6 function, ant-4/+ ail6-2 plants were transformed with a smaller AIL6 genomic fragment containing 590 bp of $5^{\prime}$ sequence and $919 \mathrm{bp}$ of $3^{\prime}$ sequence (AIL6m:gAIL6-3) (Fig. 1a). Of the 14 transgenic lines obtained, six lines conferred partial rescue of ant-4 ail6-2, five lines conferred a full rescue of ant-4 ail6-2 to the ant-4 phenotype, and four lines had an over-rescue phenotype with larger petals and some stamen anthers with four locules. This range of phenotypes closely parallels that of AIL6:gAIL6-3' lines and once again is likely a consequence of variation in transgene insertion sites. Flowers for one rescued line, AIL6m:gAIL6-3' ant-4 ail6-2 line 46, and one over-rescue line, AIL6m:gAIL6-3' ant-4 ail62 line 31, are shown in Fig. 4a-d. Floral organ counts for AIL6m:gAIL6-3' ant-4 ail6-2 line 46 are similar to ant4 and those for AIL6m:gAIL6-3' ant-4 ail6-2 line 31 

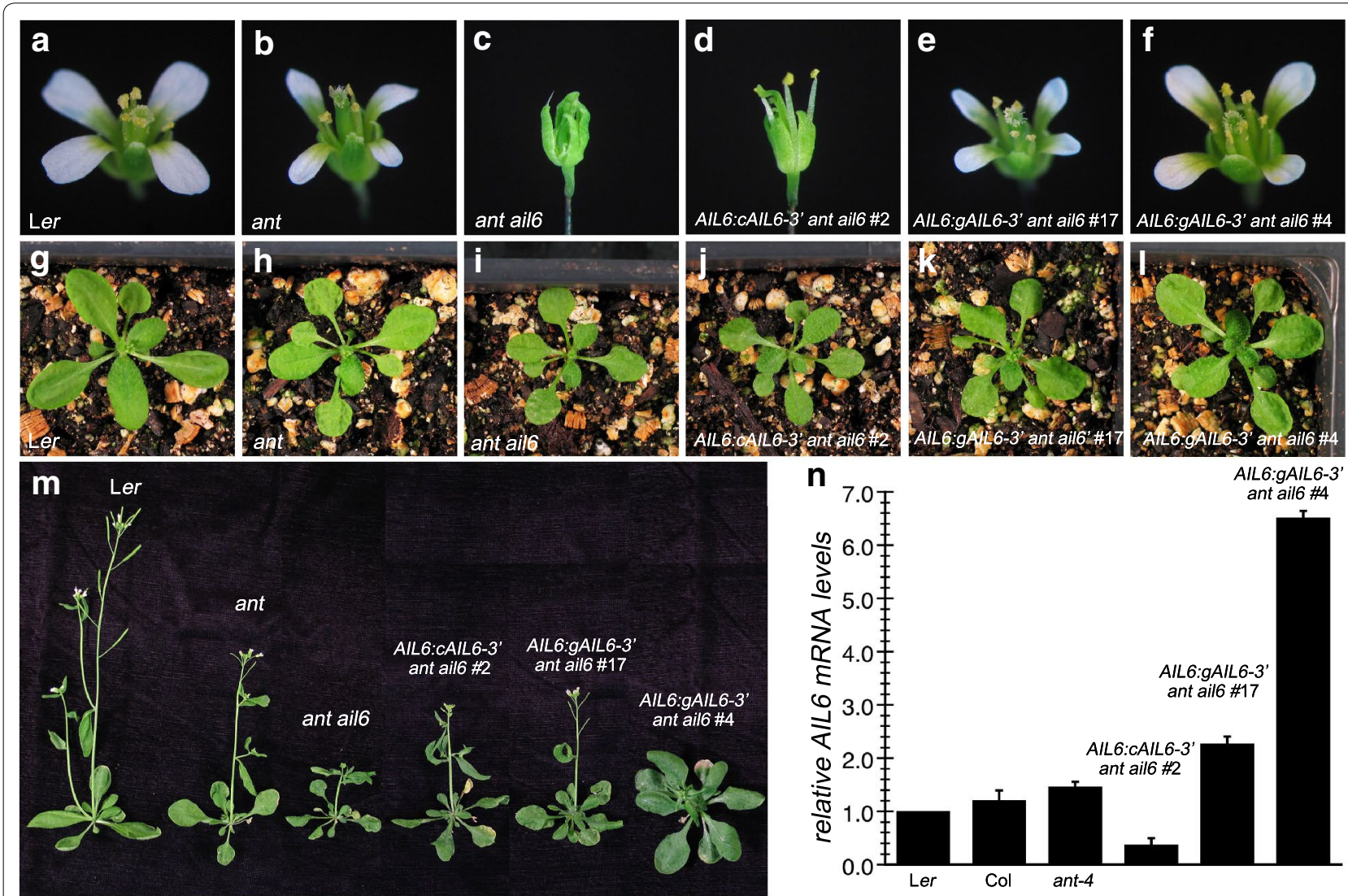

Fig. 2 a Genomic copy of All 6 with $7.7 \mathrm{~kb}$ of $5^{\prime}$ and 919 of $3^{\prime}$ sequence rescues ant ail6. Flowers from Ler (a), ant-4 (b), ant-4 ail6-2 (c), All6:CAlL6-3' ant ail6 line 2 (d), AlL6:gAlL6-3' ant ail6 line 17 (e), AlL6:gAlL6-3' ant ail6 line 4 (f). 20 day old plants corresponding to the following genotypes: Ler

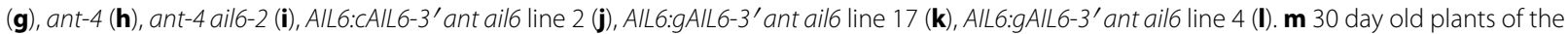
indicated genotypes. $\mathbf{n}$ Graph of RT-qPCR results showing relative AlL6 mRNA levels in Ler, Col, ant-4, AlL6:CAlL6-3' ant ail6 line 2 and AlL6:gAlL6-3' ant ail6 lines 17 and 4. Expression in Ler is set to one and the error bars show standard deviation

Table 1 Floral organ counts of genotypes utilized in this study

\begin{tabular}{|c|c|c|c|c|c|c|c|c|}
\hline & Ler & ant-4 & ant-4 ail6-2 & $\begin{array}{l}\text { AIL6:CAIL6-3' } \\
\text { line } 2\end{array}$ & $\begin{array}{l}\text { AIL6:gAIL6-3' } \\
\text { line } 17\end{array}$ & $\begin{array}{l}\text { AIL6:gAIL6-3' } \\
\text { line } 4\end{array}$ & $\begin{array}{l}\text { AlL6m:gAlL6-3' } \\
\text { line } 46\end{array}$ & $\begin{array}{l}\text { AlL6m:gAIL6-3' } \\
\text { line } 31\end{array}$ \\
\hline Se & 4.0 & 4.01 & 4.23 & 4.03 & 4.0 & 4.0 & 4.0 & 4.0 \\
\hline $\mathrm{Pe} / \mathrm{Se}$ & & 0.01 & & 0.01 & & & & \\
\hline Other flat green & & & 0.14 & 0.05 & & & & \\
\hline $\mathrm{Pe}$ & 4.02 & 3.78 & & & 3.77 & 3.30 & 3.97 & 3.99 \\
\hline $\mathrm{Pe} / \mathrm{St}$ & 0.01 & 0.01 & & & & & & \\
\hline fil & & 0.03 & 0.45 & 0.78 & 0.05 & 0.02 & 0.01 & 0.02 \\
\hline Flat St-like & & & 0.22 & 0.09 & & & & \\
\hline St-like fil & & & 0.29 & 0.84 & & & & \\
\hline St & 5.90 & 4.44 & 0.07 & 2.48 & 4.5 & 5.07 & 4.88 & 5.56 \\
\hline St-like valve & & & 0.15 & 0.03 & & & & \\
\hline Ca valve & & & 1.75 & 1.40 & & & & \\
\hline $\mathrm{Ca}$ & 2.0 & 2.0 & & & 1.99 & 2.0 & 2.0 & 2.0 \\
\hline Central fil & & & 0.23 & 0.08 & & & & \\
\hline
\end{tabular}

Se sepal, Pe/Se petaloid sepal, Pe petal, Pe/St petaloid stamen, St stamen, Ca carpel, fil filament 


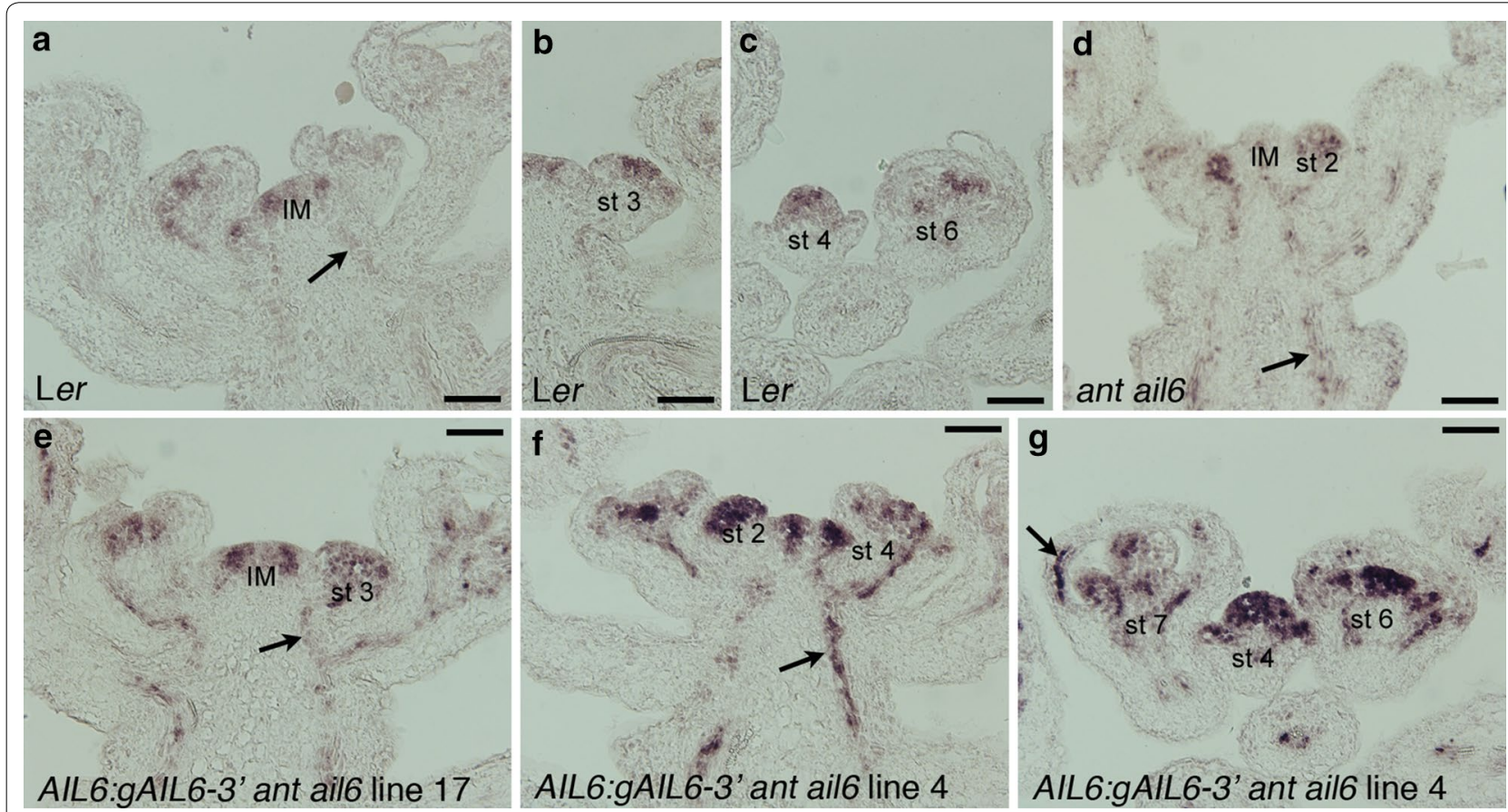

Fig. 3 In situ hybridization of AlL6 mRNA expression in AlL6:gAlL6-3'ant ail6. AlL6 expression in Ler inflorescence (a), Ler stage 3 flower (b), Ler stage 4 and 6 flowers (c), ant-4 ail6-2 inflorescence (d), AlL6:gAlL6-3' ant ail6 line 17 inflorescence, and AlL6:gAlL6-3' ant ail6 line 4 inflorescences (e, f). Arrows point to staining in the procambium in $\mathbf{a}, \mathbf{d}-\mathbf{f}$ and in a developing sepal in $\mathbf{g}$. Size bars correspond to $50 \mu \mathrm{m}$. The images in $\mathbf{a}-\mathbf{c}$ and $\mathbf{e}-\mathbf{g}$ are from slides in the same experiment and exposed to substrate for the same length of time. The image in $\mathbf{d}$ is from a different experiment. IM inflorescence meristem, st 2 stage 2 flower, st 3 stage 3 flower, st 4 stage 4 flower, st 6 stage 6 flower and st 7 stage 7 flower

approach the numbers of floral organs in wild-type flowers (Table 1). These results indicate that $590 \mathrm{bp}$ of $A I L 65^{\prime}$ region is sufficient for rescue of AIL6 in the ant-4 ail6-2 double mutant background.

To investigate whether this amount of AIL6 $5^{\prime}$ sequence was sufficient for normal AIL6 expression in flowers, the gene encoding the reporter $\beta$-glucuronidase (GUS) was fused in frame to AIL6 in the AIL6m:gAIL6-3' context (i.e. AIL6m:gAIL6-GUS-3'; Fig. 1b) was made. Of 16 transgenic lines, GUS activity was detected in young flowers of seven lines while no staining was observed in nine lines. A representative line (AIL6m:gAIL6-GUS-3' line 15) was chosen for further characterization (Fig. 4e). Examination of GUS stained and sectioned AIL6m:gAIL6-GUS-3' line 15 inflorescences under bright and dark-field illumination shows GUS activity in a pattern that matches AIL6 in situ hybridization data (Fig. 4h-k). GUS activity is detected throughout stage 1 and 2 flower primordia, becoming restricted to stamen and carpel primordia in stage 4 and 5 flowers (Fig. $4 \mathrm{~h}-\mathrm{k}$ ). These results show that this AIL6 genomic fragment is sufficient to confer a normal pattern of AIL6 expression. In contrast, no GUS activity was detected in inflorescences of any of eight lines containing an AIL6:GUS reporter in which GUS is under the control of $7.7 \mathrm{~kb}$ of AIL6 $5^{\prime}$ sequence (Figs. 1b, 4f). In addition, no GUS activity was detected in young flowers of nine AIL6m:GUS-3' lines in which GUS is present in the context of $590 \mathrm{bp}$ of $A I L 65^{\prime}$ sequence and 919 bp of AIL6 3' sequence (Figs. 1b, 4g). These results indicate that introns contain cis-regulatory elements responsible for AIL6 expression in inflorescences.

\section{The third intron of AIL6 contains enhancer elements that drive expression in young flowers}

To begin to map intronic sequences responsible for AIL6 expression in inflorescences, additional GUS reporters were made in which either intron three or intron one was placed upstream of $590 \mathrm{bp}$ of AIL6 $5^{\prime}$ sequence (Fig. 1b). These constructs also contain 919 bp of AIL6 $3^{\prime}$ sequence. Introns three and one correspond to the largest and second largest introns, respectively, within AIL6. GUS staining of inflorescences from the intron three constructs (i.e. I3F-AIL6m:GUS-3' and I3R-AIL6m:GUS-3'; Fig. 1b) showed staining throughout the inflorescence meristem and young flowers in a broader pattern than that observed in AIL6m:gAIL6-GUS-3' (compare Fig. 5a, b, e-h to Fig. $4 \mathrm{e}, \mathrm{h}-\mathrm{k}$ ). In contrast, no GUS signal was observed in the young flowers of the intron one constructs (i.e. $I 1 F$ AIL6m:GUS-3' and I1R-AIL6m:GUS-3'; Figs. 1b, 5c, d). These results suggest that intron three contains enhancer 

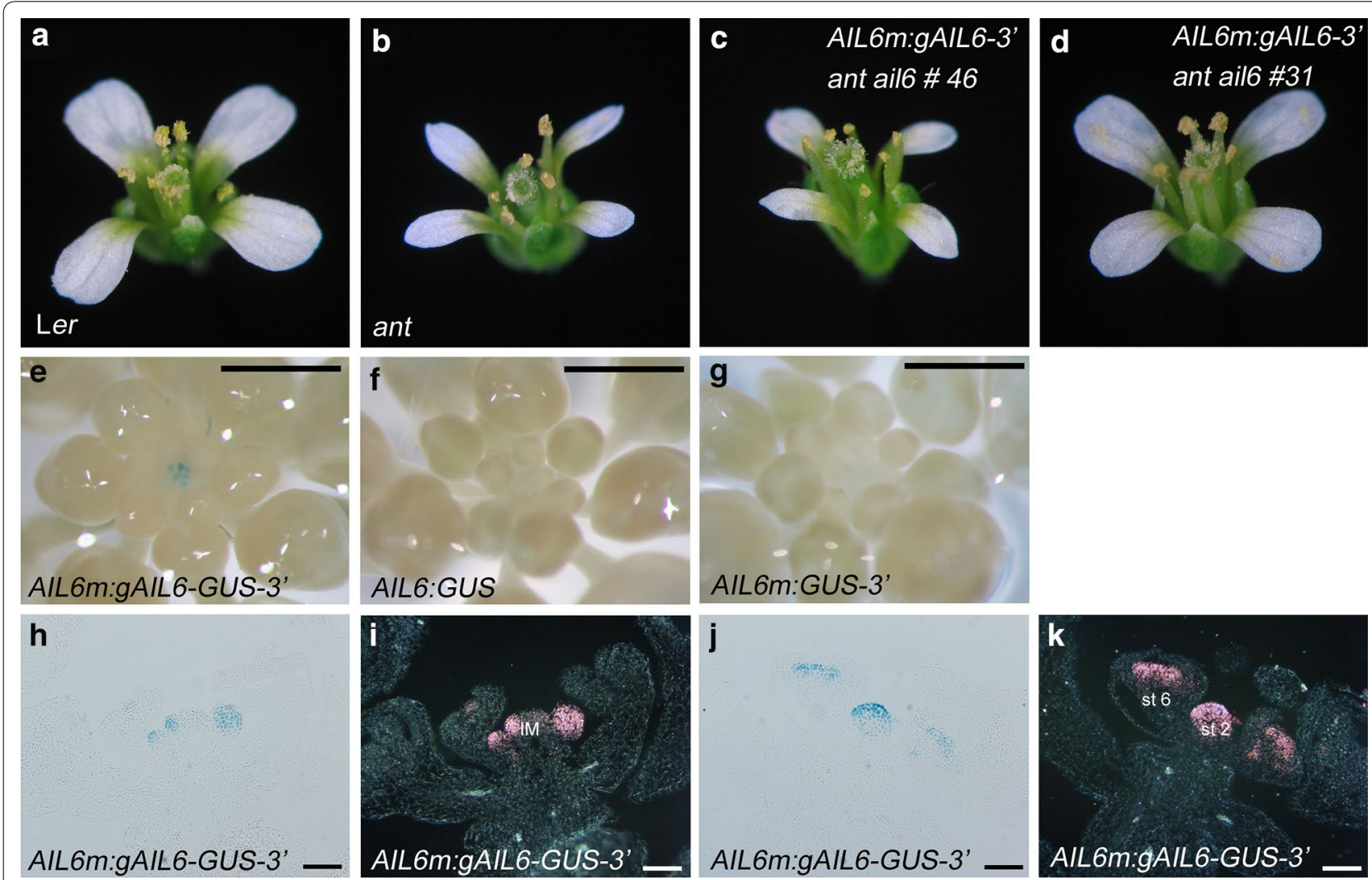

Fig. 4 A genomic copy of All 6 containing $590 \mathrm{bp}$ of $5^{\prime}$ sequence and $919 \mathrm{bp}$ of $3^{\prime}$ sequence rescues ant ail 6 and confers normal All 6 expression in inflorescences. a Ler flower. b ant-4 flower. c All6m:gAll6-3' ant ail6 line 46 flower. d All6m:gAll6-3' ant ail6 line 31 flower. e GUS stained All6m:gAll6GUS-3' inflorescence with GUS activity detected in the young flowers in the center of the inflorescence. $\mathbf{f}$ GUS stained AlL6:GUS inflorescence. No staining is visible. $\mathbf{g}$ GUS stained AlL6m:GUS-3' inflorescence. No staining is visible. h, i GUS stained and sectioned All6m:gAll6-GUS-3' inflorescence under bright field (h) and dark field (i) illumination. j, k GUS stained and sectioned AlL6m:gAlL6-GUS-3' inflorescence under bright field (j) and dark field (k) illumination. Size bars correspond to $1 \mathrm{~mm}(\mathbf{e}-\mathbf{g})$ and $50 \mu \mathrm{m}(\mathbf{h}-\mathbf{k})$. IM inflorescence meristem, st 2 stage 2 flower and st 5 stage 5 flower

elements that promote AIL6 mRNA expression in young flowers but that additional regulatory elements are present in other regions that restrict AIL6 expression within the inflorescence meristem and young flowers.

ChIP experiments have identified several transcription factors that appear to regulate AIL6 expression within inflorescences. The auxin response factor MP/ ARF5 promotes AIL6 expression in groups of cells on the periphery of the inflorescence meristem to promote flower primordium initiation [8]. MP binds to several regions of AIL6 including $5^{\prime}$ sequence, exon one, intron one, and intron three [8]. Two other putative regulators of AIL6 are the floral meristem identity proteins AP1 and LFY $[9,10]$. ChIP-Seq identified a binding peak for AP1 within intron three (Fig. 6) [9]. ChIP-chip identified a wide LFY binding region with two peaks that overlap exon two, intron two, exon three, and intron three (Fig. 6) [10]. These results are consistent with the identification of intron three as important in AIL6 regulation during early stages of flower development. Further experiments will be necessary to map additional regulatory elements that in combination with intron three confer a normal AIL6 expression pattern.

\section{Conclusions}

This study shows the importance of intronic sequences in regulating AIL6 transcription in flowers. Intron three of AIL6 is sufficient to drive expression of a reporter gene in early stages of flower development. The identification of introns as important for AIL6 regulation is consistent with ChIP data showing that several AIL6 regulators can bind to these intronic regions. In addition, increased expression of AIL6 is shown to partially compensate for loss of ANT, a gene with which AIL6 shares some functions.

\section{Methods}

\section{Plant materials and growth conditions}

Plants were grown on a soil mixture of MetroMix 360:perlite:vermiculite (5:1:1) in $16 \mathrm{~h}$ days 

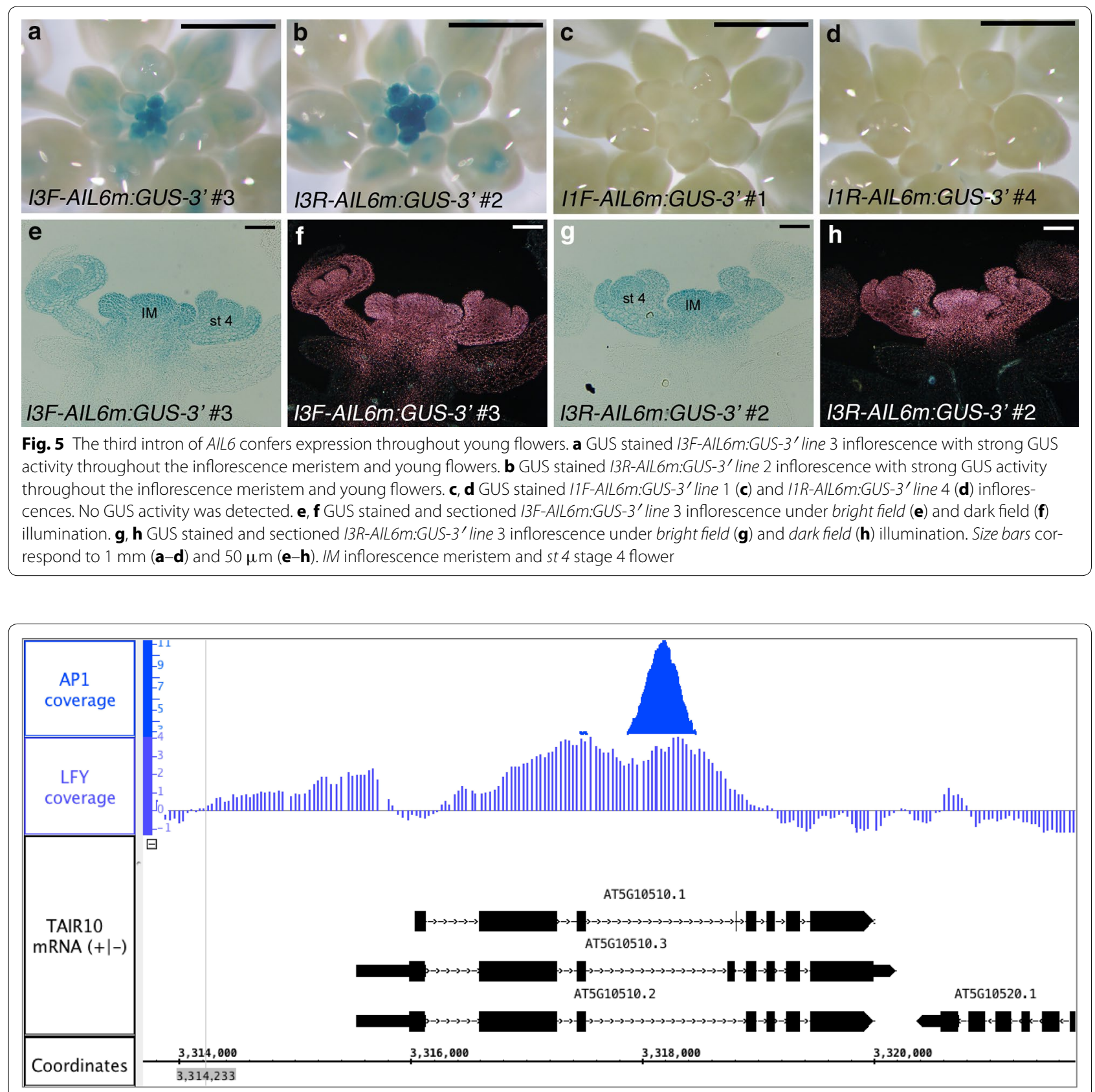

Fig. 6 ChIP data of AP1 and LFY binding to the AlL6 locus. Coverage graphs of ChIP-Seq (AP1) and ChIP-chip (LFY) data at the AlL6 locus (i.e. AT5G10510) $[9,10]$. The AP1 peak lies within intron three while the broad LFY peak overlaps exon two, intron two, exon three and intron three. This figure was created using Integrated Genome Browser (IGB) [16]

$\left(100-150 \mu \mathrm{mol} / \mathrm{m}^{2} / \mathrm{s}\right)$ at $22{ }^{\circ} \mathrm{C}$. ant -4 was PCR genotyped as described previously [4]. ant-4 ail6-2 double mutants, described previously [4], contain the strong ant-4 allele from Landsberg erecta (Ler) and ail6-2 from Columbia (Col). ant-4 contains a single T-to-A transversion that alters the splice site of the fourth intron, and ail6-2 contains a T-DNA within the third intron.
Plasmid construction and plant transformation

AIL6:gAIL6-3' was created by digestion of BAC F12B17 with BamHI, cloning of the resulting 12,666 bp AIL6 genomic fragment, which contains 7729 bp of $5^{\prime}$ sequence and $919 \mathrm{bp}$ of $3^{\prime}$ sequence, into pBluescript (AIL6 genomic/pBS). This AIL6 genomic fragment was subcloned into pCGN1547. AIL6:CAIL6-3' was created 
by PCR amplification of AIL6 cDNA with AIL6-27 and AIL6-22 (Table 2), digestion with KpnI and BamHI, and cloning into BJ36. The cDNA corresponds to AT5G10510.1 (Fig. 6) except that 45 nucleotides at the $5^{\prime}$ end of exon one, which contains an in frame upstream ATG, was included. 919 bp of AIL6 3' sequence was PCR amplified with AIL6-31 and AIL6-32 (Table 2), digested with Bam and cloned into cAIL6/BJ36. A $7.7 \mathrm{~kb} 5^{\prime}$ fragment was made by digestion of AIL6 genomic/pBS with BamHI and AflII and addition of this fragment to a clone containing $2 \mathrm{~kb}$ of AIL6 $5^{\prime}$ sequence (AIL6Pro2/pCRScript) to create AIL6Pro7.7/pCRScript. AIL6Pro7.7 was subsequently subcloned into the PstI site of pBluescript. AIL6Pro2 kb/pCRScript was created by PCR amplification with AIL6Pro1 and AIL6Pro8 (Table 2). For AIL6:CAIL6-3', the $7.7 \mathrm{~kb}$ fragment of $5^{\prime}$ sequence was subcloned from AIL6Pro7.7/pBluescript into cAIL6/ BJ36. For construction of AIL6m:gAIL6-3', partial gAIL63'/BJ36 was constructed by digestion of AIL6 genomic/ pBS with KpnI/BamHI and subcloning of the KpnI/ BamHI fragment into BJ36. A $141 \mathrm{bp}$ piece corresponding to the first exon of AIL6 was PCR amplified with AIL627 and AIL6-1 (Table 2), digested with KpnI, and cloned into partial gAIL6-3'/BJ36 to create gAIL6-3'/BJ36. $590 \mathrm{bp}$ of $A I L 65^{\prime}$ sequence directly upstream of the start codon was PCR amplified with AIL6Pro5 and AIL6Pro8 (Table 2), cloned into pCRScript and subcloned into gAIL6-3'/BJ36 cut with PstI and partially digested with NotI. AIL6m:gAIL6-3' was subcloned into pART27 using NotI. AIL6:GUS was constructed by subcloning of $7.7 \mathrm{~kb}$ AIL6 5' sequence into the PstI site of pRITA. AIL6:GUS was subcloned into the NotI site of pMLBart. For construction of AIL6m:gAIL6-GUS-3', the genomic region of AIL6 lacking a stop codon was PCR amplified with AIL648 and AIL6-50 (Table 2) and cloned into the EcoRI/ BamHI sites of pBluescript (gAIL6nostop/pBS). GUS was added into the BamHI site of gAIL6nostop/pBS. AIL6m was subcloned into gAIL6-GUS-3'/BJ97 cut with PstI and partially digested with NotI. AIL6m:gAIL6-GUS-3' was subcloned into pART27 using NotI. For construction of AIL6m:GUS-3', AIL6m was digested from pCRScript with NotI and PstI and cloned into GUS-3'/BJ97 cut with PstI and partially digested with NotI. AIL6m:GUS-3' was subcloned into pART27 using NotI. For construction of I3F-AIL6m:GUS-3', I3R-AIL6m:GUS-3', IIFAIL6m:GUS-3', and I1R-AIL6m:GUS-3', the third or

Table 2 Primers used in this study

\begin{tabular}{|c|c|c|}
\hline Primer name & Sequence & Purpose \\
\hline AlL6-27 & $\begin{array}{l}\text { ATACGGTACCATGATGGCTCCGATGACGAACTGGTTAACGTTTTCTCTGTCACCAATGGA- } \\
\text { GATGTTGAGGTCATCTGA }\end{array}$ & Cloning \\
\hline AlL6-22 & ATTGGGATCCTTAGTAAGACTGATTAGGCCAGAG & Cloning \\
\hline AlL6-31 & AATAGGATCCAACCAATCATATAAGTTGATTGAG & Cloning \\
\hline AlL6-32 & AAGAGGATCCTCGGCTAGGAAATA & Cloning \\
\hline AlL6Pro1 & TAAAGTCTGCAGATAATATAGCATAGAATCATATAATATTT & Cloning \\
\hline AlL6Pro8 & CTGCAGAAACTTTCTTATAAAAACAATTTTAC & Cloning \\
\hline AlL6-1 & TTCGAGCTTTGGGATGTGAT & Cloning \\
\hline AlL6Pro5 & TCTCTACTGCAACTTTTGTATC & Cloning \\
\hline AlL6-48 & AATAGAATTCCCCGGGATGATGGCTCCGATGACGAAC & Cloning \\
\hline AlL6-50 & AATACTGCAGGGATCCGTAAGACTGATTAGGCCAGAG & Cloning \\
\hline AlL6-52 & AGTATTCGCGGCCGCGTATATATTTTCCATTCAGTTTTCG & Cloning of I3F-AIL6m:GUS-3' \\
\hline AlL6-53 & CTTACGACGGCCGTCTGTTGATTTTAAGCAGAGCC & Cloning of I3F-AlL6m:GUS-3' \\
\hline AlL6-54 & AGTATTCGCGGCCGCCTGTTGATTTTAAGCAGAGCC & Cloning of I3R-AIL6m:GUS-3' \\
\hline AlL6-55 & CTTACGACGGCCGTGTATATATTTTCCATTCAGTTTTCG & Cloning of I3R-AIL6m:GUS-3' \\
\hline AlL6-56 & AGTATTCGCGGCCGCGTACCCTTTTCTTTCTTCTTCTCT & Cloning of /1F-AlL6m:GUS-3' \\
\hline AlL6-57 & CTTACGACGGCCGTCTTTGAAAAACAAAAACAAAAAACATCAT & Cloning of /1F-A/L6m:GUS-3' \\
\hline AlL6-58 & AGTATTCGCGGCCGCCTTTGAAAAACAAAAACAAAAAACATCAT & Cloning of /1R-AlL6m6m:GUS-3' \\
\hline AlL6-59 & CTTACGACGGCCGTGTACCCTTTTCTTTCTTCTTCTCT & Cloning of /1R-AlL6m:GUS-3' \\
\hline RTAIL6-8 & GGGATAATAGCTGTAGGAGAGAAG & RT-qPCR \\
\hline RTAIL6-9 & TCGAGCTGCCTTATCTTCTTTG & RT-qPCR \\
\hline RTFbox-1 & TTTCGGCTGAGAGGTTCGAGT & RT-qPCR \\
\hline RTFbox-2 & GATTCCAAGACGTAAAGCAGATCAA & RT-qPCR \\
\hline AlL6-FW2 & AACTGGATCCTCGGAAGGACTCATCTTGCT & Cloning \\
\hline AlL6-RV2 & AGGTGAATTCCCCTGAACGTTGGAGTTGTT & Cloning \\
\hline
\end{tabular}


first intron of AIL6 was PCR amplified with the primers listed in Table 2 and cloned into pCRScript. The corresponding introns in the forward or reverse directions (i.e. I3F, I3R, I1F and I1R) were digested from pCRScript with NotI and EagI and cloned into AIL6m:GUS-3' partially digested with NotI. The corresponding $I 3 F$ AIL6m:GUS-3', I3R-AIL6m:GUS-3', I1F-AIL6m:GUS-3', and IIR-AIL6m:GUS-3' were then subcloned into the NotI site of pART27. Plasmids involving PCR amplification were confirmed by sequencing. Plasmids were transformed into Agrobacterium tumefaciens strain ASE by electroporation. ant-4/+ ail6-2 or Ler plants were transformed with these Agrobacterium strains by vacuum infiltration [11]. Transformants were selected for kanamycin or Basta resistance.

\section{RNA extraction and RT-qPCR}

RNA extraction from inflorescences, cDNA synthesis and qPCR reactions were performed as described previously except that primers that do not amplify an AIL6 transcript in the ail6-2 background: RTAIL6-8 and RTAIL6-9 (Table 2) were used and in some cases RNA was extracted with Trizol with cleanup and DNase treatment performed on an RNeasy column (Qiagen) [12]. Data was normalized using AT5G15710 with the primers (RTFbox-1, RTFbox-2) shown in Table 2 [13]. The data shown are the average of two biological replicates.

\section{In situ hybridization}

Inflorescences were fixed, embedded, sectioned, hybridized and washed as described previously except that a hybridization temperature of $53{ }^{\circ} \mathrm{C}$ was used [14]. The AIL6 probe was made from a template corresponding to nucleotides 497-1691 of AIL6 that was PCR amplified with AIL6-FW2 and AIL6-RV2 (Table 2) using Phusion DNA polymerase and cloned into the BamHI/EcoRI sites of pGEM3Z to create longAIL6/pGEM3Z. LongAIL6/ pGEM3Z was linearized with HindIII and transcribed with T7 RNA polymerase.

\section{Organ counts}

The first 30 flowers on five plants of each genotype were counted.

\section{GUS staining}

The GUS assays were performed as described in [15]. The tissue was incubated in $2 \mathrm{mM} 5$-bromo-4-chloro-3indolyl- $\beta$-glucuronic acid for $22 \mathrm{~h}$. After taking pictures of whole inflorescences, the tissue was embedded in paraplast, sectioned, mounted on slides and observed under bright-field and dark-field illumination.

\section{Acknowledgements}

This work was supported by National Science Foundation (NSF) Grants IOS 0922367 and 1354452. I thank José Luis Riechmann and Jose Muiño for providing the AP1 coverage file.

\section{Competing interests}

The author declares that she has no competing interests.

Received: 24 July 2015 Accepted: 1 October 2015

Published online: 12 October 2015

\section{References}

1. Nole-Wilson S, Tranby T, Krizek BA. AINTEGUMENTA-like (AIL) genes are expressed in young tissues and may specify meristematic or divisioncompetent states. Plant Mol Biol. 2005;57:613-28.

2. Horstman A, Willemsen V, Boutilier K, Heidstra R. AINTEGUMENTA-LIKE proteins: hubs in a plethora of networks. Trends Plant Sci. 2014;19:146-57.

3. Galinha C, Hofhuis H, Luijten M, Willemsen V, Blilou I, Heidstra R, Scheres B. PLETHORA proteins as dose-dependent master regulators of Arabidopsis root development. Nature. 2007;449:1053-7.

4. Krizek BA. AINTEGUMENTA and AINTEGUMENTA-LIKE6 act redundantly to regulate Arabidopsis floral growth and patterning. Plant Physiol. 2009;150:1916-29.

5. Mudunkothge JM, Krizek BA. Three Arabidopsis AIL/PLT genes act in combination to regulate shoot apical meristem function. Plant J. 2012;71:108-21.

6. Prasad K, Grigg SP, Barkoulas M, Yadav RK, Sanchez-Perez GF, Pinon V, Blilou I, Hofhuis H, Dhonukshe P, Galinha C, et al. Arabidopsis PLETHORA transcription factors control phyllotaxis. Curr Biol. 2011;21:1123-8.

7. Hofhuis H, Laskowski M, Du Y, Prasad K, Grigg S, Pinon V, Scheres B. Phyllotaxis and rhizotaxis in Arabidopsis are modified by three PLETHORA transcription factors. Curr Biol. 2013;23:956-62.

8. Yamaguchi N, Wu M-F, Winter CM, Berns MC, Nole-Wilson S, Yamaguchi A, Coupland G, Krizek BA, Wagner D. A molecular framework for auxinmediated initiation of flower primordia. Dev Cell. 2013;24:271-82.

9. Kaufmann K, Wellmer F, Muino JM, Ferrier T, Wuest SE, Kumar V, SerranoMislata A, Madueno F, Krajewski P, Meyerowitz EM, et al. Orchestration of floral initiation by APETALA1. Science. 2010;328:85-9.

10. Winter CM, Austin RS, Blanvillain-Baufumé S, Reback MA, Monniaux M, Wu M-F, Sang Y, Yamaguchi A, Yamaguchi N, Parker JE, et al. LEAFY target genes reveal floral regulatory logic, cis motifs, and a link to biotic stimulus response. Dev Cell. 2011;20:430-43.

11. Bechtold N, Ellis J, Pelletier G. In planta Agrobacterium mediated gene transfer by infiltration of adult Arabidopsis thaliana plants. CR Acad Sci Ser III Sci Vie. 1993;316:1194-9.

12. Krizek BA, Eaddy M. AINTEGUMENTA-LIKE6 regulates cellular differentiation in flowers. Plant Mol Biol. 2012;78:199-209.

13. Czechowski T, Stitt M, Altmann T, Udvardi MK, Scheible W-R. Genomewide identification and testing of superior reference genes for transcript normalization in Arabidopsis. Plant Physiol. 2005;139:5-17.

14. Krizek BA. Ectopic expression of AINTEGUMENTA in Arabidopsis plants results in increased growth of floral organs. Dev Genet. 1999;25:224-36.

15. Mudunkothge JM, Krizek BA. The GUS reporter system in flower development studies. In: Riechmann JL, Wellmer F, editors. Methods in molecular biology: flower development methods and protocols, vol. 1110. New York: Springer; 2014. p. 295-304.

16. Nicol JW, Helt GA, Blanchard SGJ, Raja A, Loraine AE. The Integrated Genome Browser: free software for distribution and exploration of genome-scale datasets. Bioinformatics. 2009;25:2730-1. 